Robin Bhattarai M.S $\mathrm{PhD}^{1}$,

Chuan Chen $\mathrm{MD}^{1}$,

Chao Feng Liang $\mathrm{MD}, \mathrm{PhD}^{1}$,

Teng chao Huang MD ${ }^{1}$,

Hui Wang $\mathrm{MD} \mathrm{PhD}^{1}$,

Cong Ling MD ${ }^{1}$, Hao Li MD ,

Ying Guo $\mathrm{MD}^{\mathrm{PhD}}{ }^{2}$

${ }^{1}$ Department of Neurosurgery, Third Affiliated Hospital of Sun Yat-Sen University

${ }^{2}$ Head of Department, Department of Neurosurgery, Third Affiliated Hospital of Sun

Yat-Sen University

Address for correspondence:

Robin Bhattarai M.S. Ph.D.

Department of Neurosurgery

Third Affiliated Hospital of Sun Yat-Sen

University,

No. 600, Tianhe Road, Guangzhou

510630, Guangdong Province, PR China

Email: robinbhattarai@hotmail.com

Date submitted : 2/4/2019

Date accepted : 3/5/2019

\section{The surgical approach for clipping anterior communicating artery Aneurysm}

Anterior communicating artery (ACoA) aneurysm, accounts for $30 \%-35 \%$ of all the aneurysm making it one of the most common intracranial aneurysms. Although the Pterional approach is considered as the safe and appropriate method in ACoA aneurysm surgery, temporalis atrophy and injury to a frontal branch of the facial nerve are few inexorable complications. With the advancement of minimally invasive surgery several modified approaches, such as the supraorbital eyebrow incision approach, the minipterional approach, the mini-supraorbital approach, and the lateral supraorbital approach has been recently introduced and has been used as an alternative.

Keywords: Aneurysm clipping, anterior communicating artery, pterional keyhole, supraorbital keyhole
A CoA aneurysm is the most common aneurysm of anterior circulation aneurysms.6,15 These -aneurysms are the most complex aneurysm of anterior circulation, because of the hemodynamics of the ACoA region. ACoA aneurysms are treated by the various approaches like interhemispheric, subfrontal, lateral supraorbital and pterional. The pterional approach is most frequently used for the ACoA aneurysm.
The detailed analysis of ACoA complex shows that factors like A1 dominancy, the anatomy of the aneurysmal neck with A1 and A2 segment, perforators and presence of other vascular anomalies are required to achieve precise clipping of the aneurysms. 3 The advances in the neuroimaging like Magnetic Resonance Angiography (MRA), 3D Computed Tomography Angiography (CTA) and 3D Digital Subtraction Angiography (DSA) 


\section{ACOM aneurysm}

demonstrate the detailed anatomy around the ACoA complex before surgery. 1

Decision making regarding the surgical approach is based on A1 dominancy, projection and according to that projections, how is the plane of both A2 vessels. Yasargil16 studied the projection as a predominant anatomical factor. Inferiorly projecting aneurysms many times adhere to the optic chiasm or nerve. The dissection of the arteries that comprises the ACoA complex, recurrent arteries of Huebner (RAH), and hypothalamic arteries from the neck of an aneurysm were all considered a source of complication. Furthermore, the premature rupture before complete dissection of the dominant A1 leads to bleeding. In these situations, proximal control of the same side of the A1 segment is the most important step, and thus the dominant A1 side is better for the approaching the aneurysm.

However, there is no such specific approach for superiorly projecting ACoA aneurysm. These type of aneurysms are buried in the interhemispheric fissure and concealing the contralateral A1/A2 junction. These aneurysms partially embedded in the contralateral gyrus rectus. Thus, craniotomy on the side of A2 anterior displacement simplifies the securing of ipsilateral dominant A1. It is difficult to handle an aneurysm behind the ipsilateral A2, particularly when an aneurysm adheres tightly to A2. So it is better to approach this type of an aneurysm on the side of posterior displacement of A2 for visualizing the ACoA complex.4

\section{Review of Literature}

\section{Embryology}

In a 40-day-old embryo, an arterial plexus gives rise to the median artery of the corpus callosum and at the age of 45 days connects the two anterior cerebral arteries (ACA). Afterward, this artery regresses or disappears.12 At the same time, the involution of the arterial plexus leads to the formation of the ACoA.

Dunker2 classified three different anatomical patterns of the ACoA. Fetal type, in which the ACoA is equivalent in diameter to the ACA segment and a large median callosal artery (McaA), is present. Transitional type, corresponding to a smaller ACoA than the ACA segment with a small McaA. Adult-type, in which the ACoA diameter is less than one-third of the ACA segment with the absence of the McaA or only the persistence of a small protrusion.

The ACoA is 2 to $3.4 \mathrm{~mm}$ in diameter (average 1.5 $\mathrm{mm}$ ). The diameter is proportional to the disparity in the diameters of the two $\mathrm{Al}$ segments. A classic single ACoA joins in only 40 to 60 percent of the anterior cerebral arteries. The artery length varies from 0.8 to $4.6 \mathrm{~mm}$. The
ACoA generally lies on the optic chiasm at the level of the lamina terminalis.

\section{Microsurgical Anatomy ${ }^{8}$}

The A1 segment begins at the terminal bifurcation of the internal carotid artery (ICA) and ends at the ACoA (Figure.1). This pre-communicating segment is also called the horizontal segment because of its flat course across the optic tract to the midline. Even though the A1 segment begins and ends at branch points, the segmental anatomy of the ACA is defined by its curvature and its relationships to the brain rather than by branch anatomy. The A2 segment or post communicating segment begins at the ACoA and follows the rostrum of the corpus callosum (CC). The A3 (precallosal) segment, curves around the anterior $\mathrm{CC}$, following the genu until the artery assumes a posterior course. The A4 (supracallosal) and A5 (postcallosal) segments continue over the anterior and posterior halves, respectively, of the body of the CC, with the vertical plane of the coronal suture dividing them. The ACA's bifurcation into the pericallosal artery (PcaA) and the callosomarginal artery (CmaA) does not define these distal segments ACoA aneurysm dissection identifies 12 arteries: ipsi- and contralateral A1 segments; ipsi- and contralateral A2 segments; the ACoA; ipsi- and contralateral RAH; ipsi- and contralateral orbitofrontal arteries; ipsi- and contralateral frontopolar arteries; and the collection of ACoA perforators. The ipsilateral A1 segment is identified at the ICA terminus and leads medially and anteriorly over the optic tract and chiasm to the ACoA. On average, eight medial lenticulostriate arteries originate from the superior surface of the A1 segment and ascend to the anterior perforated substance, subfrontal area, hypothalamus, anterior commissure, septum pellucidum, and paraolfactory structures. A1 segments are symmetric in most people (90\%) but can be asymmetric due to hypoplastic or aplastic A1 segments. Despite the suggestion by angiography, absence or true aplasia of the A1 segment is rare and a vestige can be found intraoperatively. A1 segment dominance is relevant to aneurysm formation, dome projection, side of hematoma, side of the surgical approach, and proximal control. Duplicated A1 segments are rare (2\% of patients).

$\mathrm{RAH}$ is considered the "medial-most" medial lenticulostriate artery; defining the inner border of the collection of perforators along the A1 segment (medial lenticulostriates) and M1 segment(lateral lenticulostriates). As its name implies, a RAH travels back along the A1 segment to supply the head of the caudate nucleus, the putamen, the outer segment of the globus pallidus, and the anterior limb of the internal capsule, and its injury can produce contralateral face and arm weakness and 


\section{Bhattarai et al}

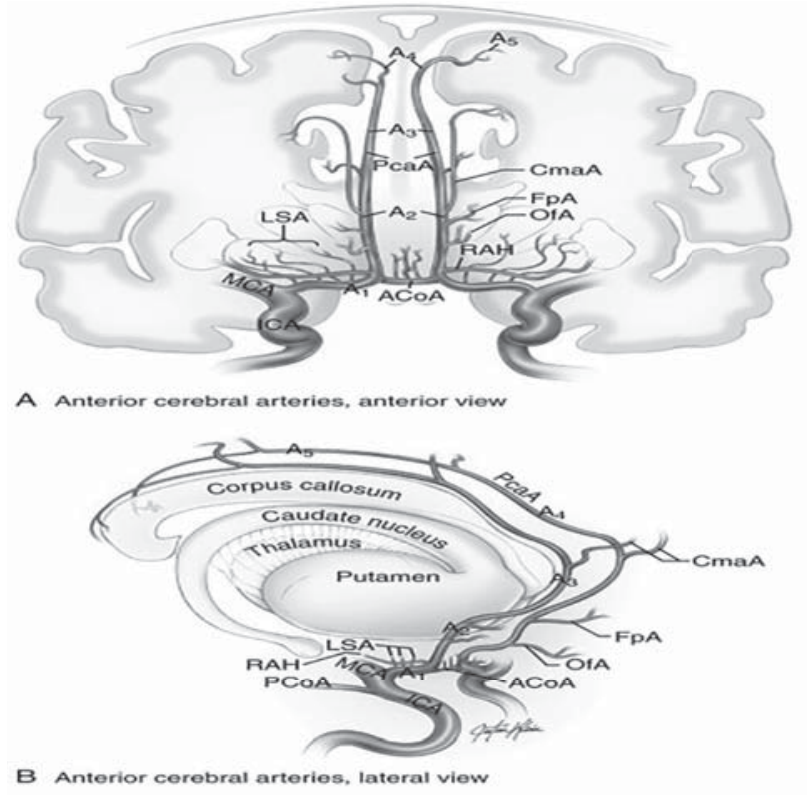

Figure 1. Microsurgical anatomy of Anterior Cerebral artery. (From Lawton, MT. Seven Aneurysms: Tenets and Techniques for Clipping. New York: Thieme, 2010.)

expressive aphasia in the dominant hemisphere. A RAH originates on the lateral wall of the proximal A2 segment just distal to the ACoA, almost as lateral continuations of the ACoA. Anatomic variation can shift its originproximal to the ACoA on the distal A1 segment, or in line with the ACoA at the A1-A2 junction, but a RAH lies within $4 \mathrm{~mm}$ of the ACoA in 95\% of patients. Exploiting this relationship in reverse, a RAH is a reliable guide to the ACoA and is particularly useful when A1 segment arcs superiorly out of view. It drapes over the shoulder of the A1 segment at its origin and lies superior (60\%) or anterior (40\%) to the A1 segment as it recurs laterally. Therefore, a RAH is often seen before the A1 segment when the frontal lobe is retracted. The artery can be duplicated in $2 \%$ of patients. The ACoA joins the A1 segments as they arrive in the interhemispheric fissure and completes the anterior circle of Willis. ACoA diameter is about half that of the A1 segments, but asymmetric A1 segments increase the ACoA diameter.

Important perforating arteries originate from the ACoA's superior and posterior surfaces and ascend to the hypothalamus, median paraolfactory nuclei, genu of CC, columns of the fornix, septum pellucidum, and anterior perforated substance. Perforators also originate from the anterior and inferior aspects of the ACoA and descend to the dorsal optic chiasm. These perforators prevent trapping or dividing the ACoA. If the anterior circle of Willis requires interruption, it is safer to divide the A1 segment, as long as the opposite A1 segment and ACoA are sufficiently large.

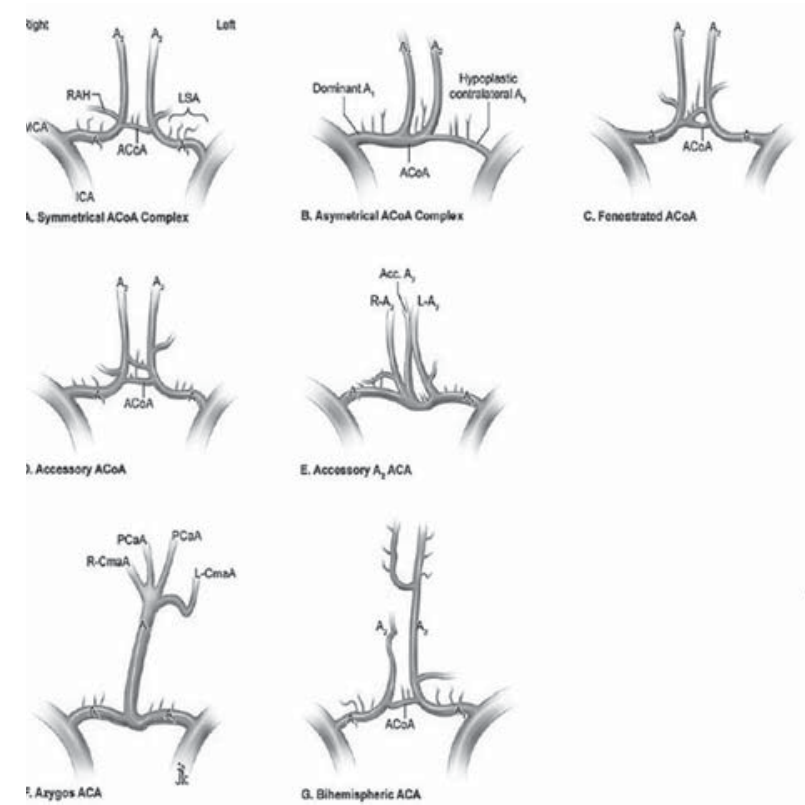

Figure 2. Normal variations of Anterior Cerebral Artery. (From Lawton, MT. Seven Aneurysms: Tenets and Techniques for Clipping. New York: Thieme, 2010.)

Orbitofrontal and frontopolar arteries lie beyond a RAH. The orbitofrontal artery is the first cortical ACA branch, arising from the anterolateral surface of the A2 segment approximately $5 \mathrm{~mm}$ distal to the ACoA and coursing perpendicularly over the gyrus rectus and olfactory tract. Its course can be similar to that of a RAH, but distally it drifts away from the A1 segment.

Orbitofrontal artery is larger in caliber than the RAH ( 2 to $3 \mathrm{~mm}$ versus $1 \mathrm{~mm}$ ). The orbitofrontal artery supplies the gyrus rectus, orbital gyri (anterior, posterior, medial, and lateral), and olfactory bulb and tract. The frontopolar artery is the second cortical ACA branch, originating from the A2 segment approximately $14 \mathrm{~mm}$ from the ACoA. Rarely, it originates from a common trunk with the orbitofrontal artery. The frontopolar artery courses anteriorly in the interhemispheric fissure and supplies the ventromedial frontal lobes.

\section{Normal variations of Anterior Cerebral artery anatomy $^{8}$}

Three variations in efferent A2 segments can lead to misinterpretations of aneurysm anatomy: azygos, bihemispheric, and accessory ACA (figure.2)

The azygos or "unpaired" ACA is a single midline artery arising from the confluence of the A1 segments and occurs in less than $2 \%$ of patients. Distally, the azygos ACA divides into the PcaA and CmaA with bifurcations, trifurcations, or quadrifurcations.

The "bihemispheric" ACA is an A2 segment that transmits branches across the midline to both hemispheres, 


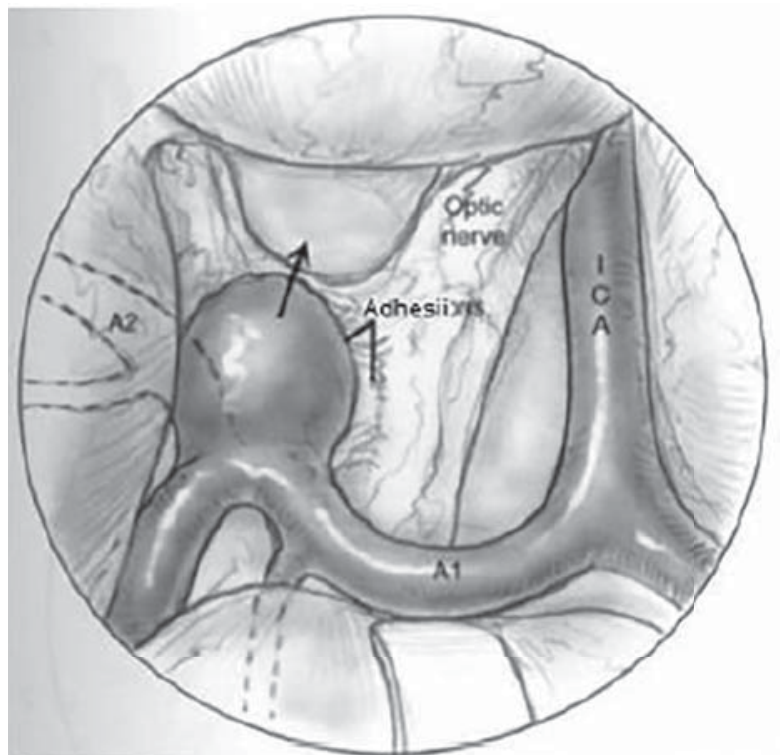

Figure 3. Inferiorly and anteriorly projecting aneurysm. ${ }^{5}$

usually in the presence of a contralateral A2 segment that is either hypoplastic or terminates early in its course toward the genu. This anomaly is seen in as many as $12 \%$ of patients.

The accessory A2 segment is a third artery originating from the ACoA in addition to ipsi-and contralateral A2 segments. The accessory ACA varies in caliber from a small remnant of the median artery of the corpus callosum (MACC) to a hyperplastic trunk resembling an azygos ACA between two smaller A2 segments. The MACC originates during embryogenesis (44days) when elongating ACA's coalesce in the midline to form plexiform anastomoses. The MACC regresses and disappears as A2 segments mature, but remnants account for the accessory ACA.

\section{Determinants of the approaching side ${ }^{7}$}

Determining factors include $\mathrm{Al}$ predominance, the direction of the A2 fork, the direction of an aneurysm, the size of an aneurysm, and the multiplicity of aneurysms. The presence of fenestration of the ACoA is an important factor in determining the side of the approach. In cases of acute stage $\mathrm{SAH}$, determining factors include the distribution of SAH and ICH. In the case of small- to large-sized aneurysms directed anteriorly (Figure.3), $\mathrm{Al}$ dominance should be the most important factor because it is sometimes difficult to secure the opposite side of Al. But there is no marked difference in surgical difficulty between the right and left approaches.

In the case of aneurysms directed superiorly, the $\mathrm{Al}$ is bilaterally secured before approaching the aneurysm. Therefore, entry into the open part of the A2 fork (i.e., the side of A2 facing posteriorly) facilitates clipping (Figure.4).
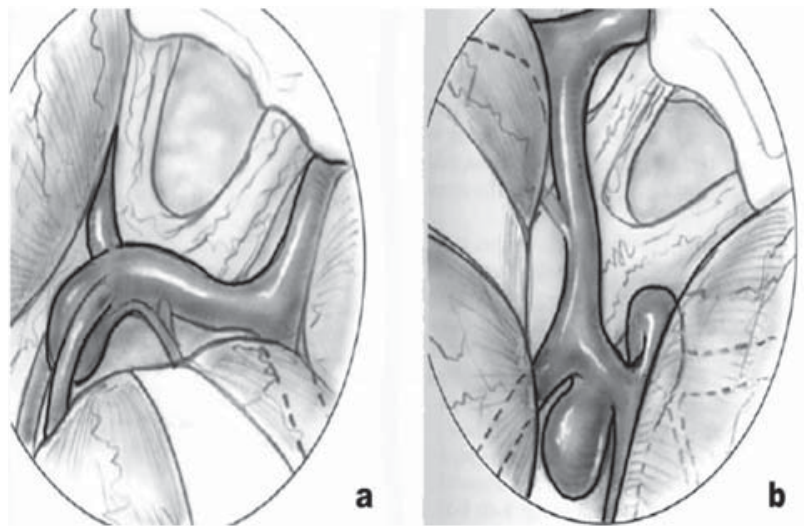

Figure 4. Superiorly projecting aneurysm (a) open A2 plane (b) closed A2 plane. $^{5}$

In the case of aneurysms directed posteroinferiorly and that are located at the back of the ACoA, entry into the side of the A2 located more anteriorly is recommended, as if the posterior surface of the A2, especially in cases with fenestration of the ACoA (Figure. 5).

Giant aneurysms are, as a rule, treated by the approach from the direction in which early arrival at the aneurysmal neck is accomplished. Approaching from the side of the dominant $\mathrm{Al}$ is generally recommended, but for such an aneurysm that projects anteriorly, the interhemispheric approach is recommended. The interhemispheric approach is also recommended in high-positioned ACoA aneurysms.11

\section{Projections}

ACoA aneurysms can project at any angle in threedimensional space. Classification of these lesions is based

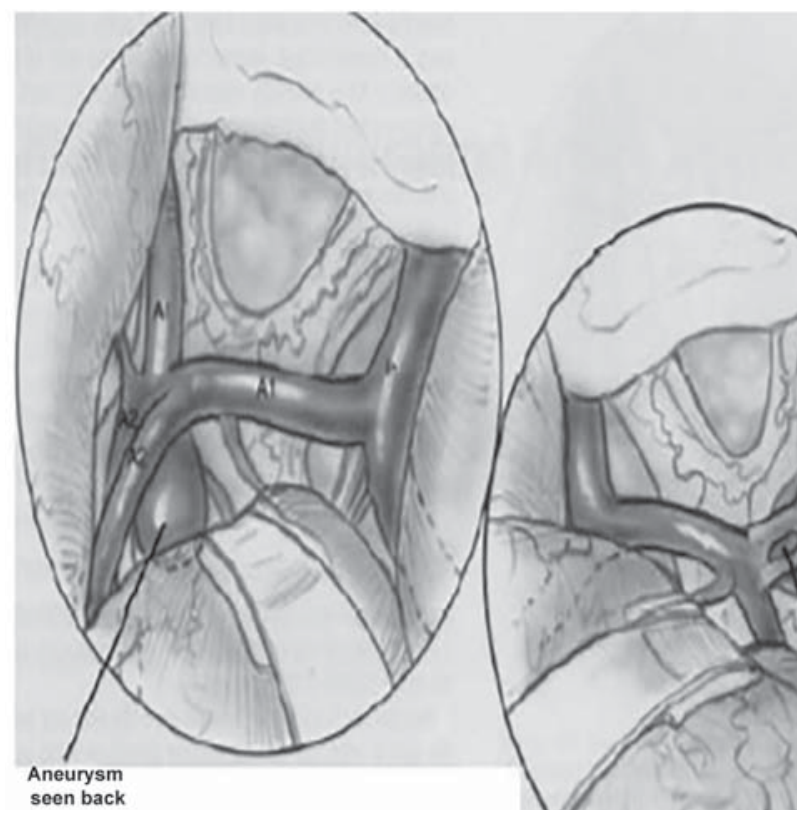

Figure 5. An aneurysm facing posteriorly combined with fenestration of ACoA. ${ }^{5}$ 


\section{Bhattarai et al}
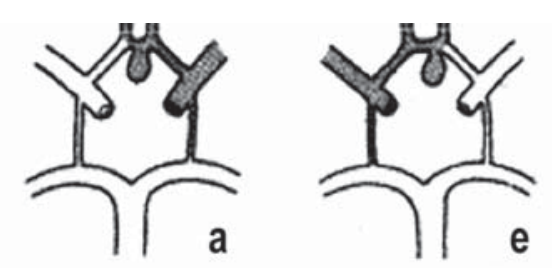

$\underbrace{1}_{b}$
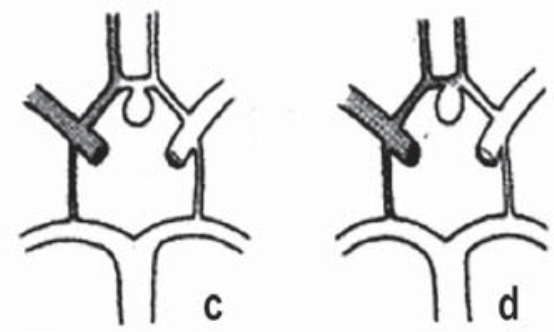

Right ACA-ACoA Aneurysm

Figure 6. Type 1: Ipsilateral Right anterior cerebral - ACoA aneurysms.

on their orientation in the true anatomical space using the optic nerves as a rough anteroposterior axis. Gary Vanderark et al.13 categorized ACoA aneurysms by the direction in which it is projected (measured as a straight line from base to fundus) into:

Superior

Anterior

Inferior

Posterior

Anterosuperior

Posteroinferior

Anteroinferior

Posterosuperior

Yasargil16 classified ACoA aneurysm projections under five groups:

Anterior

Superior

Inferior

Posterior

Complex

Nathal E et al.9 further modified it and classified projection of aneurysms into

- $\quad$ Type 1-Aneurysms located anterior to the bilateral A2 portions of the ACA

- Type 2-Aneurysms located between the bilateral A2 portions of the ACA

- Type 3-Aneurysms located posterior to the bilateral A2 portions of the ACA

Vincentelli et al.14 in their study of 60 fixed human brains indicated that all perforating branches followed a posterior direction and formed an angle with the PcaA
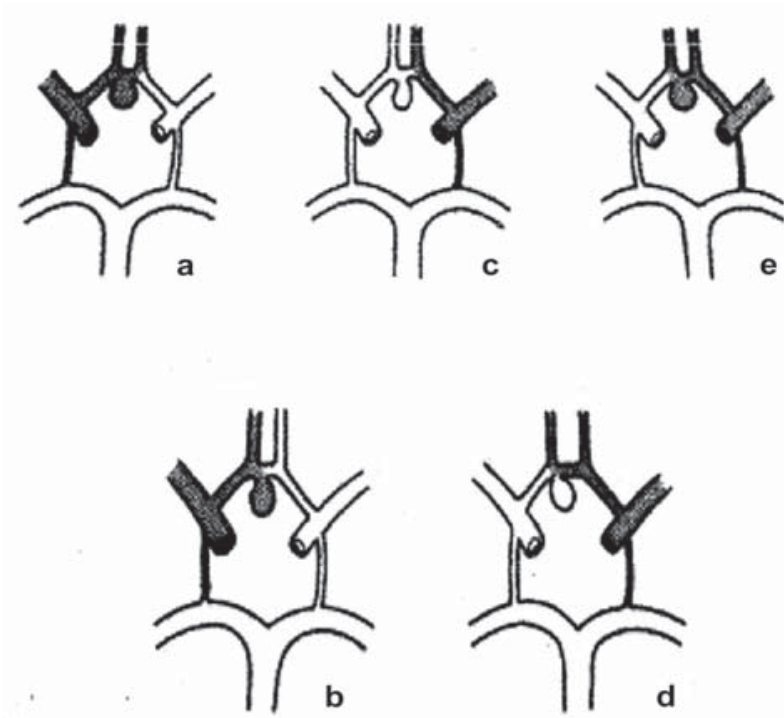

Left ACA-ACoA Aneurysm

Figure 7. Type 1: Ipsilateral left anterior cerebral ACoA aneurysms.

that ranged between 30 and 180. Yasargil16 considered this to be a predominant anatomical factor. These authors observed a decrease in positive outcomes and an increase in the mortality rate among patients with superiorly, posteriorly, and inferiorly projecting aneurysms. In these latter projections, the dissection of arteries comprising the ACoA complex, RAH, frontopolar and frontoorbital arteries and hypothalamic arteries from the neck of an aneurysm were all considered a source of complication. Conversely, for aneurysms with anterior projection, these arteries are often avoided. According to Yasargil16, different projections are

\section{Superior projection aneurysms:}

Frequently associated with a dominant ipsilateral A1 vessel, these lesions usually do not conceal the opposite optic nerve. This project into the interhemispheric fissure and the contralateral A1/ A2 junction is concealed by the aneurysmal fundus. This is themost common direction of the projection of the aneurysmal fundus and may be partially embedded in contralateral gyrus rectus. These are generally more easily handled than aneurysms projecting in other positions. Gyrus rectus resection can be helpful to mobilize the fundus. If the frontoorbital and frontopolar arteries are attached to the wall of an aneurysm it can be troublesome.

\section{Anterior projection aneurysms:}

These types fill the space between the two optic nerves, may remain within the area of lamina terminal and may be attached to the dura of anterior wall of sella turcica over tuberculum. Account for 20 to 25 percent of 

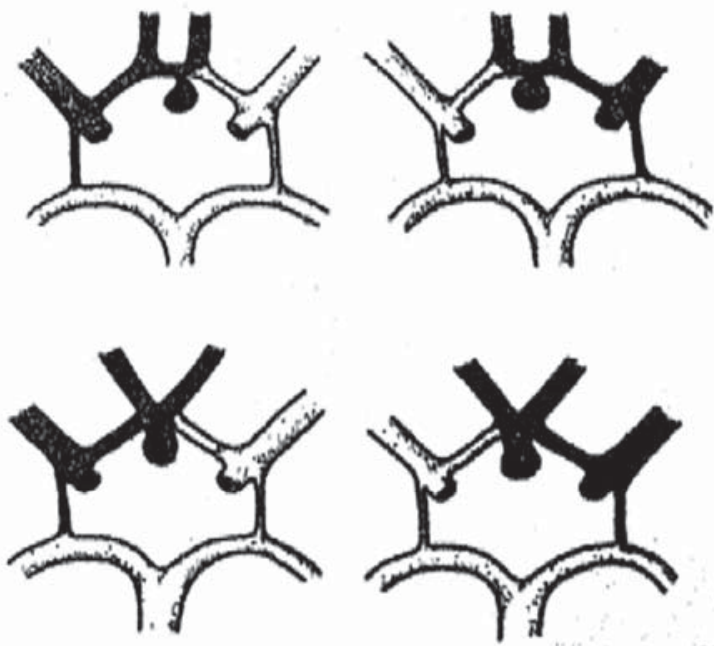

Figure 8. Bilateral ACA.

the aneurysms. They may become entangle to chiasmatic. A larger aneurysm may displace an aneurysm out of interhemispheric fissure.

\section{Posterior projection aneurysms:}

These lesions project both above and below the plane formed by the two A2 segments and usually conceal the contralateral A2 take off. Almost always involve early incision into ipsilateral gyrus rectus to visualize the anatomical structures. The chiasm is uninvolved. Fenestrated clips may be helpful for the definitive clipping of the posterior projection ACoA aneurysms.

\section{Inferior projection aneurysms:}

These projections are considered to be the most difficult to handle. The hypothalamic arteries are usually attached to the anterior wall of these aneurysms. Fundus may directly project into lamina terminalis cistern. Due to intimate association with the perforating vessels and the difficult angle for clipping, these lesions are treacherous and almost always require an extensive gyrus rectus resection to allow complete visualization of the aneurismal fundus.

\section{Complex projection:}

This aneurysm may project in any or all directions and are typically quite large with lobulations. By noticing the orientation of the fundus the surgeon will be alert to the particular problems, which may occur at the time of surgery. However, one thing worth considering and important is that there is a significant difference between the description of the aneurysm projection given by the radiologist and that actually found by a surgeon at the time of the surgery. Inferior or anterior projection aneurysms may become tethered to the optic chiasm or tuberculum
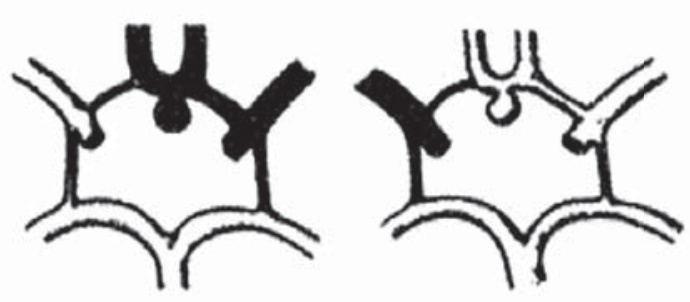

Dominant Right ACA
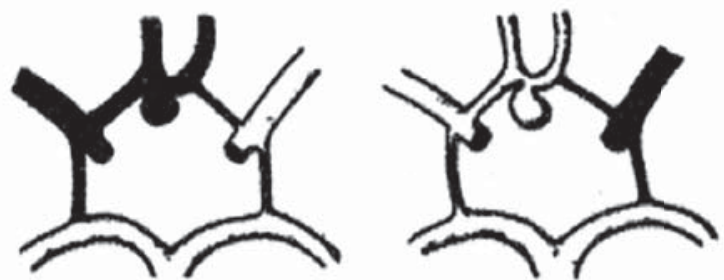

Figure 9. Type 3: Dominant ACA.

sella. A bulbous inferior or anterior directing lesion can obscure the surgeon's view of the opposite A1 segment. Retraction of the medial frontal lobe could precipitate a premature rupture of an aneurysm. A superior pointing aneurysm can become adherent to the A2, Frontoorbital or frontopolar arteries. Posterior pointing fundus can often become adherent to the perforating vessels. The surgeon must be aware of a multilobulated aneurysm. Particularly troublesome are the aneurysms with a superior and posterior projection. The surgeon may clip the superior portion of an aneurysm between the two A2 segments thereby obscuring the still patent posterior portion of an aneurysm.

Type of circulation

Norlen and Barnum14 drew attention to different patterns of circulation through the anterior part of the circle of Willis in patients with ACoA aneurysms stressing their importance for various surgical procedures. Sengupta10 noted four types of circulation, and aneurysms were classified accordingly.

Type 1: (Ipsilateral) - In this type, the aneurysm and the distal ACA filled from one proximal ACA (Figure 6).

1. Right anterior cerebral - ACoA aneurysms. The pattern of circulation in these aneurysms may be as follows

a) Right carotid injection fills an aneurysm and both the distal ACA.

b) Right carotid injection fills an aneurysm and right distal ACA.

c) Left carotid injection fills the left distal ACA only.

d) Left carotid injection with right carotid compression fills both distal ACA but not the aneurysm. 


\section{Bhattarai et al}

\section{Type IV Dominant ACA with fetal PCA}

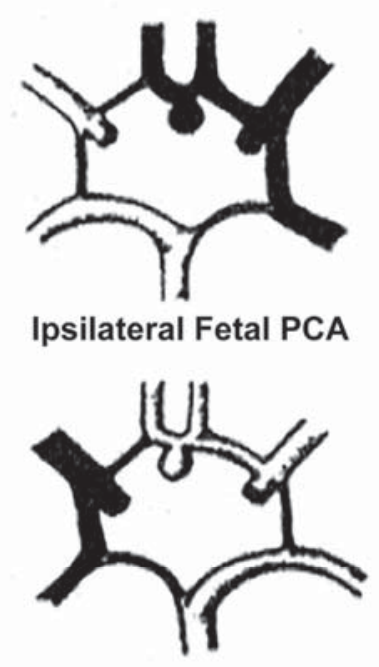

Figure 10. Dominant ACA with fetal posterior cerebral artery.

e) As in d), but an aneurysm fills.

2. Left anterior cerebral - ACoA aneurysms. In these aneurysms the following patterns of circulation are possible (Figure 7)

a) Left carotid injection fills an aneurysm and both the distal ACA.

b) Left carotid injection fills an aneurysm and the left distal ACA.

c) Right carotid injection fills the right distal ACA only.

d) Right carotid injection with left carotid compression fills both distal ACA but not the aneurysm.

e) As in d, but an aneurysm fills.

Type 2: (Bilateral) - In this type, the aneurysm and both ACA fill from both carotid injections. This pattern of circulation is often associated with rudimentary ACoA. In fact, both ACA may be fused to each other. On the other hand, the ACoA may be seen as a prominent structure on the angiograms. The significance of this analysis is that in this type of circulation the region of the ACoA receives the good collateral supply, and the effect of vasospasm is minimal. In cases of rudimentary ACoA, however, application of a clip may lead to kinking of both ACA, leading to severe ischemic problems (Figure. 8).

Type 3: (Dominant ACA) -In this type, the aneurysm arises from the axilla of the two distal ACA, both of which are divisions of the dominant ACA, and the contralateral artery is hypoplastic (Figure. 9).

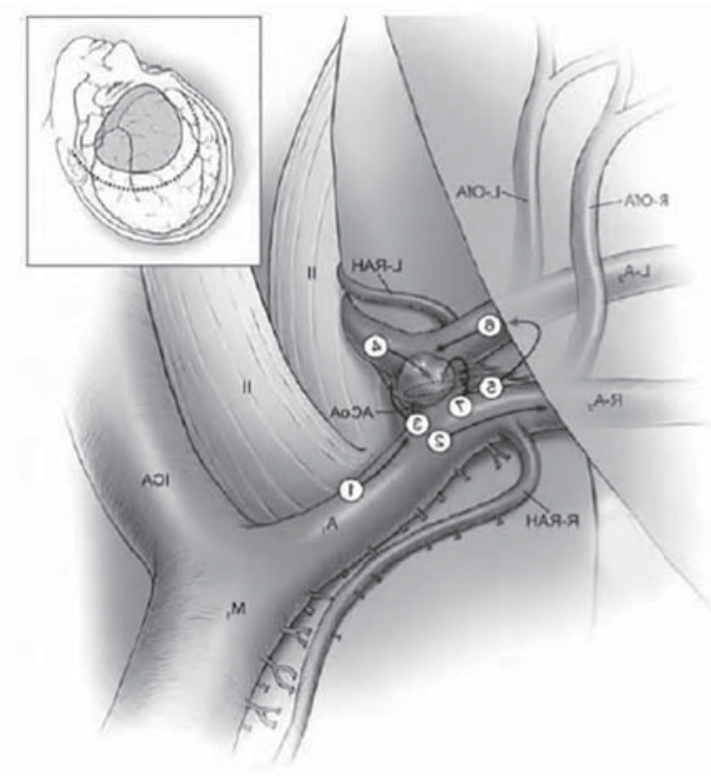

Figure 11. Aneurysm Dissection steps. (From Lawton, MT. Seven Aneurysms: Tenets and Techniques for Clipping. New York: Thieme, 2010.)

Type 4: (Dominant ACA with fetal posterior cerebral artery $\{$ PCA $\}$ ) - In this type, the circulatory patterns are like those in type 3 , but there are other associated anomalies in the circle (Figure. 10).

\section{Aneurysm dissection ${ }^{8}$}

ACoA aneurysms can be approached from either side. The right side is chosen in patients with symmetric A1 segments, and the side of the dominant A1 segment is chosen in patients with asymmetric A1 segments. This policy avoids the speech-dominant hemisphere in patients with balanced anatomy and exploits advantages of A1 dominance: early proximal control, avoidance, and favorable view of the neck. Although infrequent in the general population (around 10\%), A1 dominance is frequent in the aneurysm population (as high as 80\%). Consequently, the side of approach was evenly divided in the author's ACoA aneurysm experience. With this policy, intraparenchymal hematomas associated with rupture are typically in the contralateral frontal lobe, but are contiguous with the aneurysm dome and easily evacuated after clipping. The side of approach may be influenced by the presence of other lateral aneurysms that might be treated simultaneously. A standard pterional craniotomy is sufficient for most ACoA aneurysms, but the orbitozygomatic approach increases exposure for large, giant, and complex aneurysms (16\% in the author's experience).

The dissection of ACoA aneurysms proceeds in steps that identify the five major arteries of the dozen arteries 


\section{ACOM aneurysm}
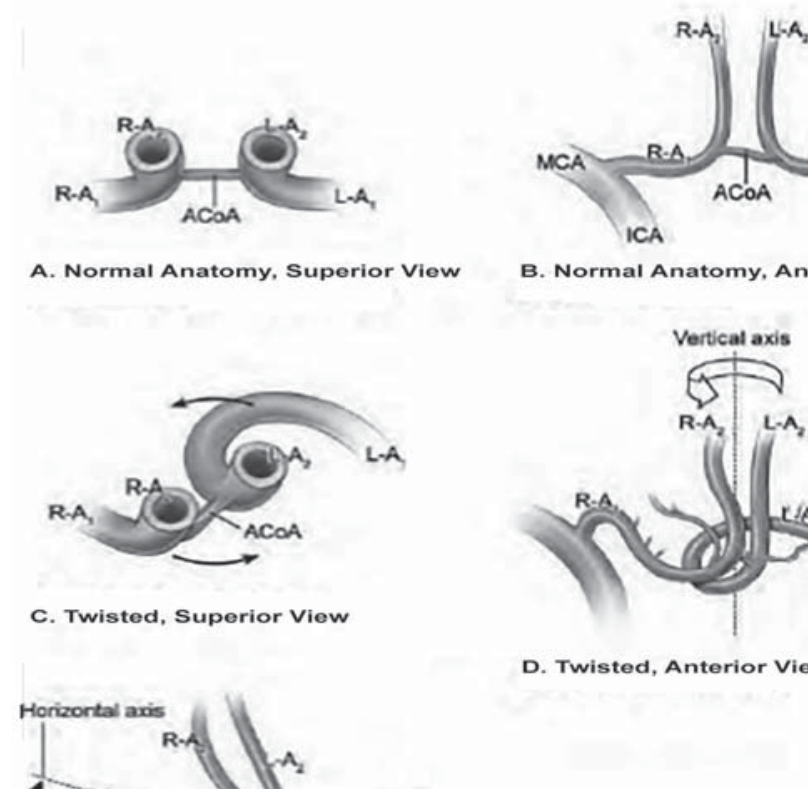

D. Twisted, Anterior Vie

Figure 12. ACoA complex and relations with major arteries. (From Lawton, MT. Seven Aneurysms: Tenets and Techniques for Clipping. New York: Thieme, 2010.)

around the ACoA complex, beginning with the ipsilateral A1 segment (Figure. 11, step 1), moving to the ipsilateral A2 segment (Figure. 11, step 2), and crossing the midline to the contralateral anatomy via the ACoA (Figure. 11, step 3). Proximal control of an aneurysm is completed by identifying the opposite A1 segment (Figure. 11, step 4). The contralateral A2 segment is located by entering the interhemispheric fissure above an aneurysm and distal to the dome (Figure. 11, step 5). The contralateral A2 segment runs parallel to the ipsilateral A2 segment and perpendicular to the contralateral orbitofrontal artery, which can often be seen anteriorly in the interhemispheric fissure. The contralateral A2 segment is traced proximally to the aneurysm neck (Figure. 11, step 6), and finally, the ACoA perforators are dissected from the posterior neck and the aneurysm is prepared for the clip blade (Figure. 11, step 7).

The ACoA complex's classic position has no lateral rotation, with the ACoA and both A2 segments lying in a coronal plane (Figure. 12, A, B); extreme rotation of the complex orients the ACoA and its A2 segments in a sagittal plane (Figure. 12, C, D). This twisting of the ACoA complex about a vertical axis shifts the A2 segments and can confuse the dissection. Dominant A1 segments tend to twist the ACoA complex to face the contralateral side in the direction of blood flow (according to Rhoton's third rule), bringing the ipsilateral A2 segment forward and making it easier to identify. However, an ACoA complex that is twisted toward the side of the approach recesses the ipsilateral A2 segment backward in the fissure and makes

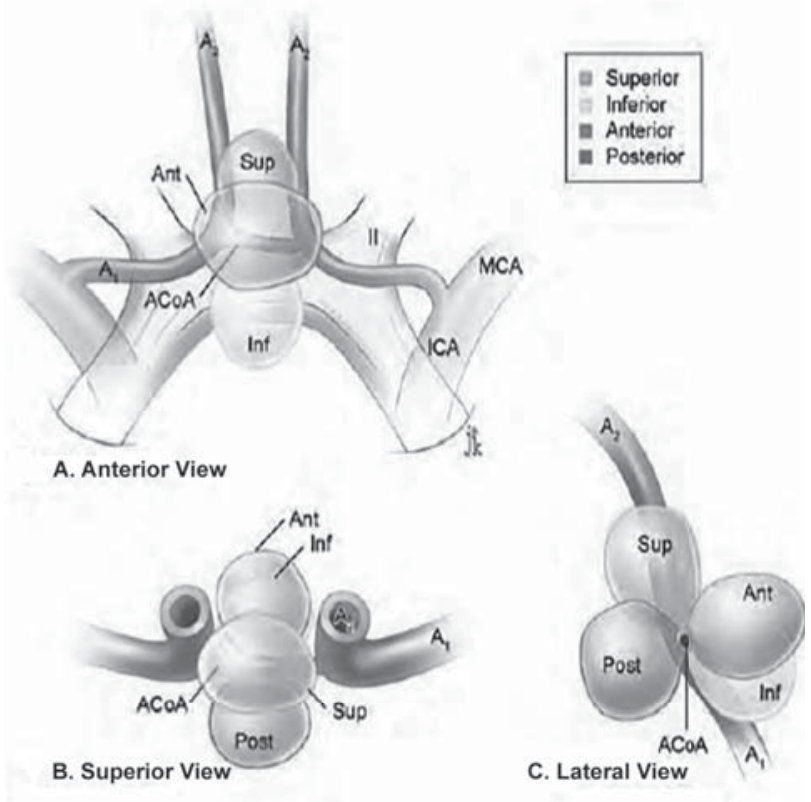

Figure 13. Dome projections of ACoA Aneurysms. (From Lawton, MT. Seven Aneurysms: Tenets and Techniques for Clipping. New York: Thieme, 2010.)

it harder to identify. In addition to this twisting rotation, the horizontal axis of the ACoA complex can also tilt (Figure. 12, E). An ACoA complex tilted downwards on the side of approach lowers the ipsilateral A2 segment and brings it into view low in the interhemispheric fissure; an ACoA complex tilted upwards on the side of approach raises the ipsilateral A2 segment and often requires gyrus rectus resection. Tilting and twisting of the ACoA complex also affect the A1 segment. The tilt toward the opposite side elevates the ipsilateral A1 segment and the superiorly arcing trajectory makes the artery more difficult to visualize.

The dissection path across the midline to the contralateral A1 and A2 segments is influenced by aneurysm projection. ACoA aneurysms project anteriorly, posteriorly, inferiorly, or superiorly (Figure. 13). In Yasargil's experience, superior aneurysms were most common (34\%), followed by anterior (23\%), posterior (14\%), and inferior (13\%); multiple lobes or mixed projection was encountered in $16 \%$ of aneurysms. Dome projection creates a surgical blind spot that conceals a critical artery or part of an aneurysm. Inferiorly projecting aneurysms hide the contralateral A1 segment, which limits proximal control. Anteriorly projecting aneurysms hide the contralateral A1-A2 junction, which hinders dissection of the distal aneurysm neck. Superiorly projecting aneurysms hide the contralateral A2 segment. Posteriorly projecting aneurysms hide the ACoA perforators, which puts them in jeopardy during permanent clipping. The dissection 


\section{Bhattarai et al}

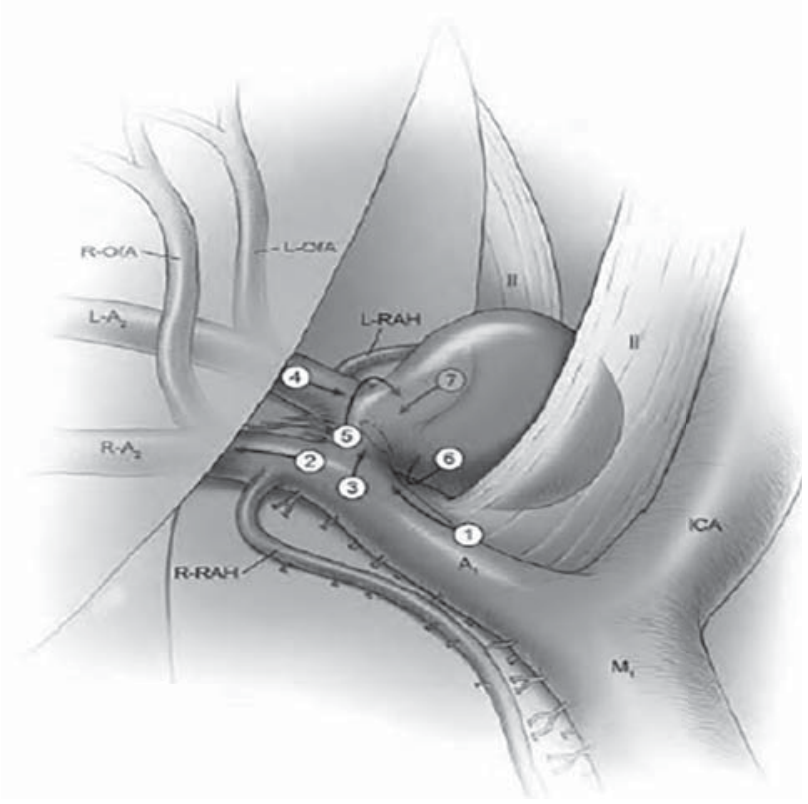

Figure 14. Dissection steps for inferiorly projecting ACoA aneurysms. (From Lawton, MT. Seven Aneurysms: Tenets and Techniques for Clipping. New York: Thieme, 2010.)

strategy is determined by the dome projection and this shifting surgical blind spot. Visible arteries in open surgical corridors are dissected first, and hidden arteries in the surgical blind spot are dissected last, often with temporary clipping, aneurysm manipulation, and some de-tethering of the dome.

With inferiorly projecting aneurysms, the contralateral A2 segment (Figure. 14, step 4) and ACoA (Figure. 14, step 5) are exposed with a superior dissection path over an aneurysm. The dome hides the contralateral A1 segment, and these aneurysms are sometimes clipped without contralateral proximal control. Full exposure of the contralateral A1 segment might require the mobilization of an aneurysm and might risk intraoperative rupture. The dome is also susceptible to avulsion with the upward mobilization of the neck when developing the plane under the neck (Figure. 14, step 6). The contralateral A1 segment and A1-A2 junction are carefully inspected after permanent clipping if they were not fully exposed before permanent clipping (Figure. 14, step 7)

With superiorly projecting aneurysms, the ACoA and contralateral A1 segment are also exposed early with an inferior dissection path (Figure. 15, steps 3 and 4). The contralateral A2 segment is hidden behind an aneurysm and cannot be found simply by ascending into the interhemispheric fissure. The initial course of the contralateral A2 segment can sometimes be seen under the

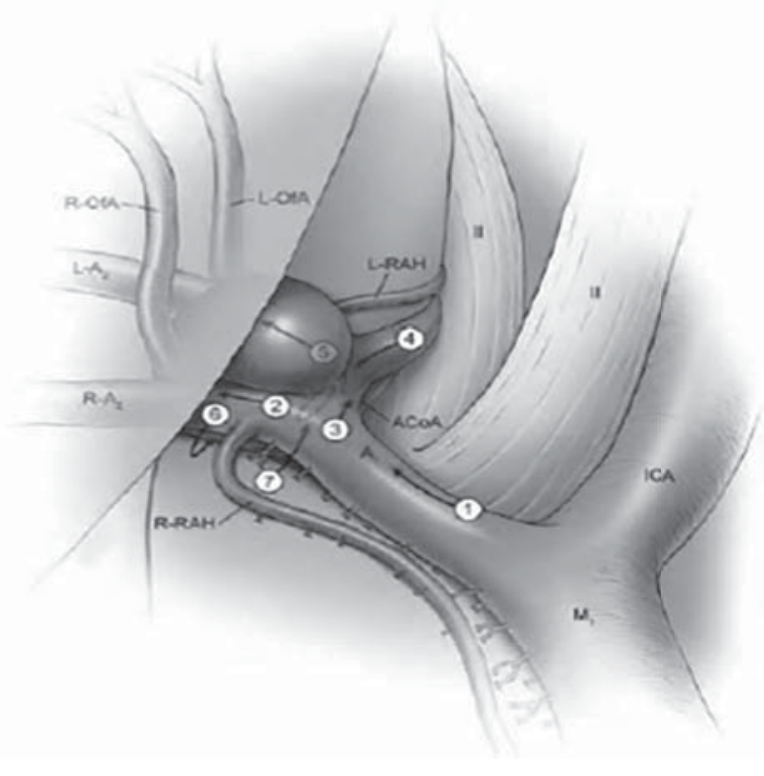

Figure 15. Dissection steps for superiorly projecting ACoA aneurysm. (From Lawton, MT. Seven Aneurysms: Tenets and Techniques for Clipping. New York: Thieme, 2010.)

belly of an aneurysm, sometimes requiring upward traction on the aneurysm (Figure. 15, step 5). However, finding the contralateral A2 ACA requires dissection behind an aneurysm, deep in the interhemispheric fissure (Figure. 15, step 6). A window is opened behind the ipsilateral A2 segment, maneuvering the artery and its orbitofrontal and frontopolar branches anteriorly. Dissection remains above a RAH; the space under it, in its axilla, is narrow, and working there can avulse this delicate artery. Superior aneurysm projection provides complete proximal control for temporary clipping and aneurysm softening, which is often needed to mobilize an aneurysm during this deep dissection. The aneurysm's posterior fundus is traversed until the contralateral A2 ACA is identified. With large aneurysms, it often helps to climb higher in the interhemispheric fissure, looking for the A2 ACA as it courses beyond the dome. When the contralateral A2 ACA is found, its inner surface is traced inferiorly, opening this important cleavage plane down to the origin of the A2 ACA and distal aneurysm neck.

The A2 origin and distal neck are carefully separated to prepare this spot for the tips of the permanent clip. This critical anatomy is right in the blind spot of the superiorly projecting aneurysm. Finally, the pathway for the posterior clip blade is opened along the neck above the posterior perforators, being careful not to over dissect delicate or densely adherent perforators (Figure 15, step 7). 


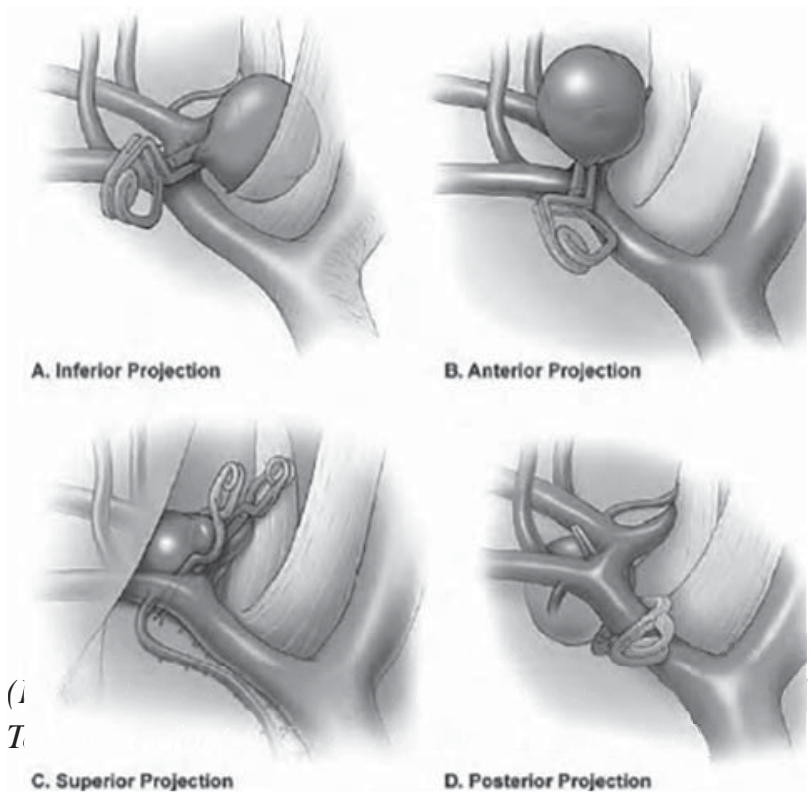

Figure 16. Clipping techniques for ACoA aneurysms. (From Lawton, MT. Seven Aneurysms: Tenets and Techniques for Clipping. New York: Thieme, 2010.)

\section{Clipping technique ${ }^{8}$}

At the end of the final dissection, Dome projection influences the clipping. Inferiorly and anteriorly projecting aneurysms are usually clipped simply with straight clips. The operative view is along the neck, with afferent and efferent branches away from the pathway of the clip blades. Inferiorly projecting aneurysms hide the contralateral A1 segment in the blind spot, and its exposure may require too much manipulation of a fragile, adherent aneurysm dome. Therefore, complete proximal control may not be available. However, the neck of an inferiorly projecting aneurysm is usually well visualized and an intraoperative rupture can be controlled by simply clipping the aneurysm. The clip's tips are inspected safely after permanent clipping; they must not pass beyond the neck and compromise the contralateral A1 afferent artery. With anteriorly projecting aneurysms, the clip's tips must not pass beyond the neck and compromise the origin of contralateral A2 ACA in the blind spot (Figure 16).

With superiorly projecting aneurysms, the ipsilateral A2 segment can interfere with the clip trajectory. A straight clip can be maneuvered around the ipsilateral A2 segment in the forefield with smaller aneurysms and simple anatomy (Figure. 14). An anteroposterior clip trajectory, with the clip facing the front of an aneurysm, avoids the ipsilateral A2 segment. The initial clip obliterates all the aneurysm behind the A2 segments, and additional under stacked clips obliterate any a remaining aneurysm in front of the
A2 segments. Wide-necked and dolichoectatic aneurysms do not permit this anterior clip trajectory because clipping often kinks the efferent arteries. Straight fenestrated clips may be more favorable, with the fenestration encircling the ipsilateral A2 ACA and the blade paralleling the ACoA (Figure. 15). Fenestrated clips are also indicated when the cleavage plane between the ipsilateral A2 segment and the proximal neck cannot be opened to pass a blade. Stacked fenestrated clips build an antegrade fenestration tube that effectively reconstructs the proximal neck without over dissecting this plane, which can be adherent to thinned aneurysm wall Posteriorly projecting aneurysms are challenging to clip.

Eleven of the 12 arteries around the ACoA complex are visible in front of an aneurysm, but the dome projects away from the neurosurgeon and displaces perforators deep in the interhemispheric fissure. The aneurysm's axis is parallel to the line of sight and the neck is behind the ACoA. This aneurysm geometry and an interposed ACoA complex often require the use of angled fenestrated clips, which are less maneuverable than straight fenestrated clips. These clips might encircle the ipsilateral A1 segment, ipsilateral A2 segment, and/or the ACoA itself, and the view of the perforators is limited. These concerns are exacerbated by perforators that adhere to an aneurysm.

\section{Conclusion}

ACoA aneurysms are the most complex aneurysm of anterior circulation. The detailed analysis of ACoA complex shows that factors like A1 dominancy, the anatomy of the aneurysmal neck with A1 and A2 segment, perforators and presence of other vascular anomalies are required to achieve precise clipping of the aneurysms.
Abbreviations and Acronyms
ACA: Anterior cerebral arteries
ACoA: Anterior communicating artery aneurysm
CC: Corpus callosum
CmaA: Callosomarginal artery
CTA: Computed Tomography Angiography
DSA: Digital Subtraction Angiography
ICA: Internal carotid artery
ICH: Intracerebral hemorrhage
MACC: Median artery of the corpus callosum
McaA: Median callosal artery
MRA: Magnetic Resonance Angiography
PcaA: Pericallosal artery
PCA: Posterior cerebral artery
RAH: Recurrent arteries of Huebner
SAH: Subarachnoid hemorrhage 


\section{Bhattarai et al}

\section{Reference}

1. Avci E, Fossett D, Aslan M, Attar A, Egemen N. Branches of the anterior cerebral artery near the anterior communicating artery complex: an anatomic study and surgical perspective. Neurol Med Chir 43:329-333, 2003.

2. Dunker RO, Harris AB. Surgical anatomy of the proximal anterior cerebral artery. J Neurosurg 44:359-367, 1976

3. Fox JL. Craniotomy for aneurysm, cranial approaches, in Fox JL(ed): Intracranial aneurysms. New York: Springer, Vol 2, pp750-799, 1983

4. Hino A, Fuse I, Echigo T. Clipping of upward projecting anterior communicating aneurysms via pterional craniotomy: approach from the side of A2 of anterior displacement. No Shinkei Geka 34:14958, 2006

5. Hirotoshi S. Anterior communicating artery aneurysms surgical approaches review, in Laligam N.Sekhar, Fessler R (eds): Atlas of Neurosurgical Techniques. New York: Thieme, pp 142-152, 2006

6. Kassell NF, Torner JC, Haley EC, Jane JA, Adams HP, Kongable GL. The international cooperative study on the timing of aneurysm surgery. J Neurosurg 73:18-36, 1990

7. Kato Y, Yamada Y, Nouri M. Anterior Communicating Artery Aneurysms, in Laligam N.Sekhar, Fessler R (eds): Atlas of Neurosurgical Techniques. New York: Thieme, pp 162-171, 2016

8. Lawton TM. Anterior communicating artery aneurysms, in Conerly K (ed): Seven Aneurysms
Tenets and Techniques for Clipping. New York: Thieme, pp 94-120, 2011

9. Nathal E, Yasui N, Sampei T, Suzuki A. Intraoperative anatomical studies in patients with aneurysms of the anterior communicating artery complex. J Neurosurg 76:629-634, 1992

10. Pasqualin A, Bazzan A, Cavazzani P, Scienza R, Licata C, Pian RD. Intracranial hematomas following aneurysmal rupture: Experience with 309 cases. Surg Neurol 25:6-17, 1986

11. Sekhar LN, Natarajan SK, Britz GW, Ghodke B. Microsurgicalmanagementofanteriorcommunicating artery aneurysms. Operative Neurosurgery 61:273292, 2007

12. Truwit CL. Embryology of the cerebral vasculature. NeuroimagG Clin N Am 4: 663-89, 1994.

13. Vanderark GD, Kempe LC. Classification of anterior communicating aneurysms as a basis for surgical approach. J Neurosurg 32:300-303, 1970

14. Vincentelli F, Lehman G, Caruso G, Grisoli F, Rabehanta P, Gouaze A. Extracerebral course of the perforating branches of the anterior communicating artery: Microsurgical anatomical study. Surg Neurol 35:98-104, 1991

15. Yasargil MG. Anterior cerebral and anterior communicating artery aneurysms, in Yasargil MG (ed): Microneurosurgery, New York: Georg Thieme Verlag Vol 2, pp 180-185, 1984

16. Yasargil MG, For JL, Ray W. The operative approaches to aneurysms of the anterior communicating artery, in Krayenbuhl $\mathrm{H}$ (ed): Advances and Technical Standards in Neurosurgery. New York: Georg Thieme Verlag, Vol 2, pp 114-170, 1975 